\title{
Caracterización de configuraciones subjetivas de éxito en directivos intermedios del sector turístico*
}

\author{
Characterization of success subjective configurations in intermediate \\ managers of tourist industry
}

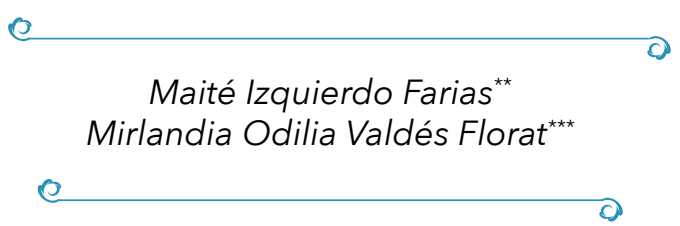

Recibido 15. 05.2019 • Arbitrado 20.07. 2019 •

Aprobado 22.08. 2019

El artículo presenta resultados de un estudio que responde al Proyecto institucional: Formación y desarrollo de competencias laborales en el sector turístico de Camagüey. Universidad de Camagüey.

* Máster en Ciencias de la Educación otorgado por la Universidad de Camagüey. Departamento de Psicología-Sociología de la Universidad de Camagüey. Ciudad de Camagüey, Cuba. ORCID: 0000-0003-4749-1938, maite.izquierdo@reduc.edu.cu

*** Doctora en Ciencias Pedagógicas otorgado por la Universidad de Camagüey. CEDET, Universidad de Camagüey. Ciudad de Camagüey, Cuba.mirlandia.valdes@reduc.edu.cu

\section{Resumen}

La acción de dirigir se enriquece en la misma medida en que aumenta la complejidad de las tareas de dirección, en este proceso el directivo se forma, al tiempo que se estructuran las configuraciones subjetivas que condicionan el éxito de sus funciones. La presente investigación permite caracterizar las configuraciones subjetivas de éxito en directivos intermedios del sector turístico para el ejercicio de sus funciones de dirección. En el proceso de darle cumplimiento al objetivo se asume una metodología cualitativa y el método de estudio de caso, se aplicaron las técnicas: entrevistas en profundidad y completamiento de frases. Los resultados develan la construcción de significados y sentidos subjetivos por parte de los sujetos estudiados, en cuanto a su rol como directivos, que se expresan en el amor por su trabajo, la influencia de este en su vida familiar y en su 
comportamiento, etc.; y que están en constante actualización, en relación con las exigencias y demandas de la organización y el medio social circundante.

Palabras claves: configuraciones subjetivas, sentidos subjetivos, dirección.

\section{Abstract}

Managing action is enriched in the same way that management tasks complexity increase, the manager is trained in this process at the same time subjective configurations are structured that determine his function's success. The current research allows to characterize success subjective configurations in intermediate managers of tourist industry for the practice of their managing functions. In the process of giving accomplishment to the research objective it is assumed a qualitative methodology and the case study method, they were applied the techniques: interviews in-depth and phrases fill in. Results show the construction of meanings and subjective senses by the studied subjects, in regard to their role as managers, that are expressed in the love for their job, the influence of this one in their family life and behavior, etc.; and these are in constant update, in relation with organization exigencies and demands and surrounding social medium.

Keywords: subjective configurations, subjective senses, management.

\section{Introducción}

Las organizaciones laborales coexisten en contextos de acelerado desarrollo en el orden económico, social y tecnológico. Esto impone a las empresas el desafío de actualización, superación y resiliencia para alcanzar resultados eficaces y eficientes en su gestión. Las organizaciones cubanas no escapan de esta situación actual, especialmente las turísticas que deben mantener determinados niveles de competitividad, lo que depende en gran medida de los directivos responsables.

Las entidades del sector turístico en Cuba cuentan con recursos naturales, humanos y de capital, así como con los factores tanto externos como internos que pueden contribuir al éxito de sus servicios. De ahí que el rol de sus directivos resulte una actividad muy importante en la articulación y aplicación de un conjunto de principios, normas y técnicas para mejorar y optimizar el uso de estos recursos.

Los directivos en el sector turístico deben conjugar todos aquellos valores individuales con las exigencias del cargo para de esta forma obtener éxito. 
Sobre todo, aquellos directivos cuyas funciones se orientan a la supervisión de los servicios que tienen como objetivo la satisfacción del cliente.

Los estudios (López y Martínez, 2014; Ramírez, Guerra y Ramis, 2016; López, Reyes y Molina, 2017) planean la importancia de los indicadores intangibles en la gestión directiva de las organizaciones turísticas, haciendo referencia al componente socio-emocional, la inteligencia emocional, la satisfacción con el empleo, la motivación de los trabajadores, las competencias, etc. Cada uno de estos indicadores se basa en la individualidad del sujeto que dirige y en su experiencia personal. Sin embargo, estos estudios no profundizan en la base subjetiva que atraviesa la acción diaria del directivo, y que, al mismo tiempo, permite la construcción de significados y sentidos que condicionan su éxito.

La acción directiva está mediada por procesos internos de carácter subjetivo que están en constante actualización en relación con las exigencias y demandas de la organización y el medio social circundante: las configuraciones subjetivas, las cuales encuentran en el espacio laboral un contexto de progreso desde diferentes aristas, así como también su expresión en la regulación y autorregulación del comportamiento en la organización, todo lo cual conlleva en su máxima expresión a mejores niveles de eficiencia y eficacia.

En las organizaciones laborales los procesos se estructuran y organizan producto de la acción directiva de uno o varios hombres. Sus funciones típicas se enmarcan en la planificación, organización, regulación y control de todos los procesos, ejecutadas desde el ejercicio de la autoridad, liderazgo, comunicación, motivación y supervisión. Sobre estos hombres pesa el desempeño de un papel muy importante, porque de ellos depende el éxito o fracaso de las empresas que dirigen.

Autores como Hernández y Rodríguez (2006) y Ruiz (2012), al definir la dirección, contemplan elementos que dependen del actuar del directivo y que pueden o no estar presentes en las características personológicas de los mismos en el momento de ocupar el cargo. Sin embargo, el propio ejercicio de las funciones de dirección propicia una relación de desarrollo que permite que el directivo vaya perfilándose en el cargo.

Esto es posible por la configuración subjetiva de la acción que se organiza en el curso de la experiencia. Esa configuración subjetiva de la acción, según González Rey (2013), es, a su vez, inseparable de la configuración subjetiva de la personalidad que anticipa los estados y disposiciones subjetivas que definen a la persona al entrar en la acción. 
Las organizaciones laborales marcan la vida del hombre, pues en estos contextos se conforman y se expresan aspectos subjetivos que le imprimen un sello de peculiaridad a la relación que se establece entre ambos. De manera que el hombre trabajador nutre su personalidad con cada experiencia laboral que vivencia y esto conlleva a la construcción de sentidos subjetivos que orientan el comportamiento del sujeto en la organización.

Se entretejen así, elementos históricos y culturales a los modos de pensar, sentir y actuar del hombre como sujeto organizacional que no está exento de los vínculos con la sociedad. El comportamiento de este responde a sentidos sociales, como consecuencia del intercambio interpersonal de significados y del desarrollo de necesidades cualitativamente superiores (Vistorte, 2011).

De acuerdo con lo anterior, toda nueva experiencia del hombre en la organización puede comprenderse como una producción de sentido y no como reflejo de la realidad. Aquellas particularidades de su subjetividad individual le propician una interrelación productiva con el medio laboral. Esto le permite alcanzar mayor integración entre su subjetividad y la realidad social con la cual interactúa, podrá actualizar y atribuir, en mayor medida, sentidos psicológicos a esa realidad.

La subjetividad es un espacio de constitución de la psiquis humana e individual en los diversos momentos de relación del ser humano, con los diversos estados, procesos y sentidos subjetivos que sea capaz de conferirles. Por lo que se expresa tanto a nivel individual como social, como constituyente de esa vida social e individual, o sea, en los espacios donde el hombre sea capaz de asignar un sentido a la realidad objetiva (González Rey, 2010).

La actuación del sujeto, según González Rey (2013), siempre ocurre dentro de redes de subjetividad social donde los otros, así como los diferentes efectos de sus acciones e interrelaciones, están siempre presentes en la configuración subjetiva de la acción individual. El sujeto es un elemento esencial de toda producción social, pues su acción es generadora de nuevos momentos de subjetivación social, de los que pueden emerger verdaderas alternativas de funcionamiento social.

Las configuraciones subjetivas expresan cierta movilidad. El sujeto no es capaz de modificarlas de manera brusca sino mediante un proceso donde se transforman paulatinamente las representaciones, creencias y vivencias que posibilitan su enfrentamiento a representaciones ya conformadas (Vistorte, 2011). Es decir, estas no tienen un carácter estático, por el contrario, son históricos e individuales. 
Como se ha dicho anteriormente la organización laboral constituye un espacio de desarrollo individual para el hombre trabajador, es en este contexto donde el sujeto pasa la mayor parte de su día a día. Esta representa una de las redes de subjetividad social más relevantes en la vida del individuo, generadora de elementos de sentidos subjetivos que se configuran de forma múltiple, tanto a nivel individual como en la organización de la vida social.

Lo expuesto hasta el momento ofrece elementos que permiten deducir la necesidad de profundizar en el estudio del hombre como sujeto en las organizaciones laborales, con énfasis en los aspectos subjetivos que intervienen en esta relación. Esta investigación en particular se centra en los directivos de un nivel intermedio en instituciones hoteleras, quienes alcanzan resultados exitosos en el desempeño de sus funciones, lo cual es visible y reconocido a través de indicadores tangibles relacionados con la proyección estratégica. Sin embargo, este éxito alcanzado no es relacionado con los elementos de subjetividad que intervienen en el actuar de estos directivos.

La configuración subjetiva del ejercicio de la dirección, desde los propios sujetos que la ejercen, ha sido escasamente tratada en estudios científicos relevantes (Maceo y Montero, 2017). Lo cual respalda la importancia de su estudio para una mayor comprensión y acercamiento al factor humano en el área de la dirección específicamente en el sector del turismo.

Unido a lo anterior, en un estudio del año 2016 en instituciones hoteleras, los autores Ramírez, Guerra y Ramis, en sus resultados constatan la necesidad de mejorar la calidad percibida en la atención al cliente, potenciando la experiencia positiva de este, que se construye esencialmente en el marco de la relación profesional que se establece al momento de brindar el servicio, donde es el factor subjetivo un elemento esencial. Intervienen en estos procesos fundamentalmente el personal que labora en la gastronomía (restaurant, cafetería, bares, etc.), cuyas actividades son planificadas, organizadas y supervisadas por un directivo especialista en el área que es conocido como Mailtre, cargo intermedio de dirección en un hotel.

Todo lo hasta aquí expuesto motiva la presente investigación a orientar su atención a la caracterización de las configuraciones subjetivas de éxito en directivos intermedios del sector turístico relacionados con el ejercicio de sus funciones de dirección. 


\section{Metodología}

La presente investigación se realizó sobre la base de una metodología cualitativa la cual facilitó un acercamiento a los participantes en su ambiente natural de trabajo y en relación con el propio contexto laboral. Permitió profundizar en sus puntos de vistas, interpretaciones y significados.

La investigación tuvo lugar a través del método de estudio de caso. Se trabajó con una muestra de expertos, constituida por 3 mailtres (cargo intermedio de dirección en un hotel), pues se consideró que estos eran los participantes idóneos para ofrecer información sobre el éxito en la dirección de procesos que garantizan la satisfacción del cliente.

Se aplicaron las técnicas de entrevista en profundidad y completamiento de frases.

La entrevista en profundidad se realizó con el objetivo de explorar las unidades de sentido que configuran las proyecciones de los sujetos en la vida individual y organizacional. Se tomaron entre algunos temas a profundizar: historia de vida personal, historia de vida laboral, elementos de sentido relacionados a situaciones laborales, específicamente de dirección, etc.

El completamiento de frases realizado fue una técnica cuyas frases fueron elaboradas por las autoras para ajustarlo a los intereses del estudio. El objetivo de esta técnica se centra en analizar las proyecciones que expresa el sujeto sobre el proceso de dirección, su vida laboral y el éxito de sus funciones.

El estudio fue realizado bajo consentimiento informado y preservando la identidad de sus participantes.

La información obtenida fue procesada a través del método de análisis de contenido por medio del cual se analizó la frecuencia de aparición de varios indicadores. Esto permitió determinar unidades de análisis y arribar a la triangulación de los resultados.

\section{Resultados y discusión}

Para este estudio se tomaron como escenarios los hoteles de ciudad pertenecientes a la cadena Isla Azul. La investigación se centró en el área de alimentos y bebidas $(\mathrm{A}+\mathrm{B})$ por tratarse del área que más espacios de interacción tiene con el cliente y una de las cuales posee mayor responsabilidad en el éxito de las instituciones hoteleras. Se trabajó con los directivos principales de esta área en cuestión, para un total de tres participantes, todas mujeres, 
con un rango de edad de 35 a 50 años y una trayectoria laboral entre los 7 y 15 años de experiencia en la rama del turismo y la dirección.

El procesamiento de los resultados se realizó a través del análisis de contenido lo que permitió identificar dos unidades de análisis fundamentales: familia, trabajo de dirección. Los resultados se presentarán según estas unidades.

\section{Unidad de análisis: familia.}

En las tres participantes del estudio, la familia ha significado y significa mucho para su desempeño personal y profesional. Esta es un área en la que fue posible identificar varias unidades de sentidos subjetivos: en relación con los padres, con los hijos, y con la pareja.

Los logros de sus carreras profesionales están ligados a la orientación y guía de las figuras paternas: “...mis padres siempre me apoyaron en todo..."; “... gracias a las exigencias de mis padres he logrado muchas cosas, se los agradezco siempre..."; “...celebran mis triunfos como si fueran de ellos...". El papel orientador de los padres, unido al vínculo afectivo-emocional sustenta el sentido de la acción, en donde intervienen las motivaciones y las necesidades de estos sujetos.

Pareja e hijos constituyen elementos fundamentales de la mayoría de los proyectos de vida de un hombre o una mujer, una vez alcanzados, atraviesan todas las esferas de la vida. Para las participantes de esta investigación, estos son pilares fundamentales. La pareja el sostén ante las dificultades, el impulso para asumir y alcanzar metas: “...mi esposo me ayuda mucho...”; “... para mi es importante saber que puedo contar con él...". En el caso de los hijos refieren: "...mis hijos, por ellos hago todo..."; "...aunque este cansada de tanto trabajo estar con ellos me hace sentir mejor...". Ciertamente el rol de madres representa un agente movilizador del comportamiento para las participantes del estudio, pues más allá de su responsabilidad social con las exigencias laborales, sus hijos impulsan su desempeño exitoso en esta esfera. Representan una motivación muy fuerte cuyo origen principal se centra en la satisfacción de sus necesidades.

Se pudo comprobar que para las participantes de la investigación la familia de origen, como la familia conformada, representan unidades de sentidos subjetivos que tienen una naturaleza configuracional. Cada una de ellas marca sus experiencias en todos los contextos y condiciona su comportamiento. Al respecto refieren: “...la unión de la familia es lo más importante...”; “...con mi familia cerca disfruto más mis logros...”. 
Un aspecto importante a mencionar en esta unidad de análisis es que se contrapone en cierta medida a la esfera laboral, las participantes en muchas ocasiones refieren sentirse divididas por los intereses y motivaciones que emergen de una y otra (familia y trabajo). Refieren que por el tipo de responsabilidad que desempeñan sus funciones en la familia se ven un tanto afectadas: “...me pierdo muchos momentos importantes de mi familia por cumplir con el trabajo..."; "...mis responsabilidades en el trabajo me alejan un poco de mis deberes como esposa...". Ambos aspectos son esferas importantes para las participantes del estudio, incorporan sentidos vivenciales a su existencia y constituyen objetivos fundamentales de su proyecto de vida. Familia y trabajo son generadores de sentidos subjetivos que orientan la acción personal de estas y tienen una naturaleza configuracional, y en este caso, la presencia de estos como unidades de sentidos en estrecha relación e interrelación, forman parte de un mecanismo motivacional que las encamina al éxito de sus funciones de dirección.

\section{Unidad de análisis: trabajo de dirección.}

Los sujetos de esta investigación poseen una amplia trayectoria como directivos, y sienten satisfacción por lo que hacen: “...me encanta lo que hago, el turismo es algo muy bonito... me gusta porque está directamente en áreas de atención al cliente"; “...me siento realizada, paso el día aprovechando la jornada laboral, desde que llego me pongo en funciones...".

Como directivos se puede afirmar por los resultados procesados que poseen operatividad y organización en el trabajo, establecen correctamente las metas y las acciones para alcanzar las mismas: "para lograr algo se debe tener una proyección que te permite idear las acciones. Al proyectarte te relacionas con lo que quieres lograr, te permite mejorar, perfeccionar"; "primeramente me concentro en lo que tengo que hacer, me planifico con tiempo de antelación, todo el tiempo pienso y organizo las ideas en relación al trabajo, tengo buena memoria, siempre estoy pendiente de las cosas, intento equilibrar las necesidades y las prioridades, primero va la prioridad porque es más apremiante". De manera que dirigir para estas participantes más que una función es un proceso, un estilo de vida que aplican diariamente y a través del cual han adquirido competencias para hacerlo y al mismo tiempo han perfeccionado su trabajo.

Como directivo se relacionan adecuadamente con sus subordinados, tratando de combinar su rol con sus características propias de personalidad: “...con mis compañeros soy humanista, con un grado de exigencia de mi para ellos, y hay de ellos para mí un respeto que lo considero importante, soy exigente con un 
alto grado de compañerismo, con ellos y con cualquiera, tenemos un $A B C$ que no podemos perder, que es saludar, dar los buenos días, a pesar de los problemas que se traigan de la casa"; "...soy amable y justa, pero siempre controlo, de lo contrario se acomodan, se debe exigir para evitar la indisciplina". Refieren un clima de comunicación, colaboración, y entendimiento: “...tengo muy buen equipo, pues siempre trato de convencer a las personas de porque tienen que hacer las cosas con argumentos sólidos"; “...intento que se trabaje en equipo, que exista cooperación, que funcionemos como una familia”. Configurándose dinámicamente un espacio donde se manifiestan valores de responsabilidad, solidaridad y compromiso, existe una implicación profunda y compleja, desde su articulación, por parte de la relación directivo-subordinado.

Esta unidad de análisis permite explicar que las decisiones, modo de actuación, construcciones e ideas de las participantes de la investigación son fuentes de sentido subjetivo que se integran activamente en la configuración subjetiva de su acción como directivos. El éxito de sus funciones es resultado del curso de los recursos y posiciones que van desplegando en el proceso de vivir su experiencia en el ejercicio de la dirección.

Entre los aspectos que las caracterizan en su función se pueden mencionar que favorecen la comunicación con sus subordinados: “... para lograr un buen ambiente en el trabajo se debe respetar a todo el mundo, no importa la función que realice, escuchar y decir las cosas en un tono firme pero sin perder la ternura...". La confianza (logran que sus subordinados sean capaces de confiar en ellas y, además, en ellos mismos) “... cuando se es jefe hay que aprender a conocer a todos, de esta forma logras estimular sus potencialidades y su seguridad en ellos mismos...”, “... es fundamental creer en uno mismo, si no lo hace uno los otros tampoco lo harán...”. La integridad, se relaciona fundamentalmente con la honestidad y responsabilidad y, muy especialmente, la credibilidad “... no se puede ser como dice el dicho: haz lo que yo digo y no lo que yo hago, una tiene que tener coherencia entre lo que dice y hace...”. En su desempeño intervienen factores claves como: la delegación de responsabilidades “... ser jefe no significa que todo lo haga una, está dentro de nuestra función delegar acciones y responsabilidades, eso estimula al trabajador en cierta medida...”. La motivación: “... para mí, mi trabajo es lo más grande...”. La valoración de la creatividad y esfuerzo personal, la valoración del trabajo en equipo, etc: "...las buenas ideas e iniciativas hay que reconocerlas eso estimula al trabajador, más aún cuando se pone en práctica su propuesta...”, “...lo más reconfortante es que el cliente reconozca el trabajo de un turno completo, desde cocina, bar, hasta servicio habitación, eso da muestra que están en sintonía, que hay trabajo de equipo". 
De acuerdo con todo lo analizado se puede deducir que el directivo mediante el ejercicio de la dirección experimenta un proceso de crecimiento, el cual se organiza en complicadas configuraciones subjetivas, las cuales, teniendo como base los sentidos subjetivos, le permiten establecer una relación especial con el medio laboral e interpretar la realidad objetiva. De tal forma mediante el cumplimiento de sus funciones adquiere nuevos sentidos y significados propios, o simplemente los actualiza, los externaliza y, por este medio, interactúa y transforma su entorno.

\section{Conclusiones}

La investigación permitió identificar como unidades de sentido subjetivo que intervienen en el desempeño exitoso del ejercicio directivo: la familia y el trabajo de dirección. La familia como principal portador de vivencias, motivación, sentimientos y vínculos emocionales, etc.; y el trabajo de dirección como principal escenario de dinámicas y situaciones que conducen a la configuración subjetiva de la acción individual de dirigir.

El trabajo de un directivo interviene en la dinámica familiar, y conlleva a que se contrapongan intereses familiares a laborales, ambos constituyen sentidos subjetivos que orientan la acción personal de un directivo, esta contraposición posee una naturaleza configuracional y no lineal; y en este caso encamina al éxito de las funciones de dirección.

La satisfacción por el desempeño del rol de dirección, las competencias que se desarrollan en el transcurso de la actividad directiva matizadas con las características individuales del sujeto, las relaciones directivo subordinado, etc, son fuentes de sentido subjetivo que se integran activamente en la configuración subjetiva del ejercicio de la dirección. El éxito de este es resultado del curso de los recursos y posiciones que van desplegando los directivos en el proceso de vivir su experiencia.

Los resultados obtenidos demuestran la importancia del estudio de la subjetividad en las organizaciones laborales pues toda acción del sujeto genera nuevos momentos de subjetivación social y organizacional, por lo que profundizar los estudios en este tema permitiría identificar las problemáticas presentes y su jerarquización en aras de buscar soluciones sostenibles y con un carácter humanizante. 


\section{Referencias}

González, F. (2010). Las categorías de sentido, sentido personal y sentido subjetivo en una perspectiva histórico-cultural: un camino hacia una nueva definición de subjetividad. Universitas Psychologica, 9 (1), 241-253. Obtenido de http://www. redalyc.org/articulo.oa?id $=64712156019$

González, F. (2013). La subjetividad en una perspectiva cultural-histórica: avanzando sobre un legado inconcluso. Revista Ciencias Sociales, 11, 19-42. DOI: https://doi. org/10.18046/recs.i11.1565

Hernández y Rodríguez, S. (2006). Introducción a la administración. Teoría general administrativa: origen, evolución y vanguardia. México: Mc Graw Hill. http:// online.fliphtml5.com/vfco/wiyx/\#p=3

López, R., Martínez, A. (2014). Inteligencia emocional y "La ventana del líder" en los directivos turísticos. Ingeniería Industrial, XXXV (2), 229-239. Obtenido de http://scielo.sld.cu/scielo.php?pid=S1815-59362014000200010\&script $=$ sci arttext\&tlng $=$ en

López, R., Reyes, Y., Molina, A. (2017). Evaluación del impacto de la capacitación en directivos de instalaciones turísticas. Humanidades y Ciencias Sociales, 28(54), 130-149. Obtenido de http://www.scielo.org.ar/scielo.php?pid=S1851$17162017000100005 \&$ script $=$ sci_abstract\&tlng $=$ pt

Maceo, D., Montero, D. R. (2017). Configuración subjetiva del ejercicio de la dirección en directivos. Universidad y Empresa, 19(33), 75-112. DOI: 10.12804/revistas. urosario.edu.co/empresa/a.4963

Ramírez,E., Guerra, L., Ramis, C. (2016). Trabajo emocional y satisfacción con el empleo en los hoteles de sol y playa. Retos de la dirección, 10(1), 142-161. Obtenido de http:// scielo.sld.cu/scielo.php?script $=$ sci_arttext\&pid $=$ S2306-91552016000200005

Ruiz, P. G. (2012). Dirección. Obtenido de Red tercer milenio: https://www.srh.de/ fileadmin/Websites/Holding/General/Pdf/DIRECCION.pdf

Vistorte, Y. (2011). Configuración subjetiva de la calidad de los servicios en las organizaciones laborales. Contribuciones a las Ciencias Sociales, julio 2011, Obtenido de www.eumed.net/rev/cccss/13/ 